\title{
Polarized generalized parton distributions and structure functions of the $\rho$ meson
}

\author{
Bao-Dong Sun ${ }^{1,2, *}$ and Yu-Bing Dong ${ }^{1,2,3}$ \\ ${ }^{1}$ Institute of High Energy Physics, Chinese Academy of Sciences, \\ Beijing 100049, People's Republic of China \\ ${ }^{2}$ School of Physics, University of Chinese Academy of Sciences, \\ Beijing 100049, People's Republic of China \\ ${ }^{3}$ Theoretical Physics Center for Science Facilities (TPCSF), \\ CAS, Beijing 100049, People's Republic of China
}

(Received 8 November 2018; published 31 January 2019)

\begin{abstract}
The $\rho$ meson polarized generalized parton distribution functions, its structure functions $g_{1}$ and $g_{2}$ and its axial form factors $\tilde{G}_{1,2}$ are studied based on a light-front quark model for the first time. Comparing our obtained moments of $g_{1}$ to the lattice QCD calculation, we find that our results are reasonably consistent to the lattice predictions.
\end{abstract}

DOI: $10.1103 /$ PhysRevD.99.016023

\section{INTRODUCTION}

It is believed that the generalized parton distributions (GPDs) of a system could be a powerful tool to understand its hadronic structure [1]. This is because GPDs naturally embody the information of both form factors (FFs) and parton distribution functions (PDFs) for the complicated system. They can provide the normal PDFs for the longitudinal parton distribution as well as the transverse information. Consequentially, GPDs display the unique properties to present a "three-dimensional (3D)" description for the transverse and longitudinal partonic degrees of freedom inside the system. Furthermore, it should be addressed that the physical meaning of the transverse distribution is more transparent when one goes to the impact parameter space [2-4]. Another important potential of GPDs is the information about how the orbital angular momentum contributes to the total spin of a hadron. We know that the sum rules proposed by Xiangdong Ji for a nucleon (spin-1/2) reveal the relation between GPDs and the spin carried by quarks and gluons $[5,6]$. For the spin- 1 hadrons, such as deuteron and $\rho$ meson, one may also reach similar relations. Meanwhile, they provide some new structure functions which have no analogue to the case of spin-1/2 targets [7-9].

For a spin- 1 target, there are 9 helicity nonflip GPDs and 9 helicity flip GPDs for each quark flavor (or for the gluon)

\footnotetext{
*sunbd@ihep.ac.cn
}

Published by the American Physical Society under the terms of the Creative Commons Attribution 4.0 International license. Further distribution of this work must maintain attribution to the author(s) and the published article's title, journal citation, and DOI. Funded by SCOAP. at the twist-2 order. The spin-1 helicity nonflip (twist-2) GPDs are defined in Ref. [8] by considering the deeply virtual Compton scattering and meson electroproduction processes of the deuteron. Recently, the 9 helicity flip (twist-2) GPDs, or transversity GPDs, are introduced and discussed in Ref. [10]. Among the total 9 helicity nonflip quark GPDs, 5 of them are unpolarized and 4 of them are polarized ones. The sum rules of the unpolarized GPDs can give the charge $G_{C}$, magnetic $G_{M}$, and quadrupole $G_{Q}$ form factors. We have intensively studied those observables with a help of a light-front constituent quark model for the $\rho$ meson phenomenologically [11], where the $\rho$ meson form factors $G_{C, M, Q}\left(Q^{2}\right)$, mean square charge radius, magnetic, and quadrupole moments are calculated. Our obtained results are reasonably compatible with the previous model calculations and the experimental data [12-14]. Moreover, our calculated results for the first Mellin moments of the unpolarized GPDs $H_{1}$ and $H_{5}$, which respectively correspond to the reduced matrix elements and to the structure functions of $F_{1}$ and $b_{1}$ (the tensor structure), are in a good agreement with the results from the lattice QCD calculation [15]. For the transversity GPDs of $\rho$ meson, they are remained to be studied. In this work, only helicity nonflip GPDs are considered.

To account for a polarized target, we know that the spindependent structure functions $g_{1}(x)$ and $g_{2}(x)$ are defined by the decomposition of the imaginary part of the forward virtual Compton scattering amplitudes [7,16-18]. In the leading order (twsit-2), the forward limit of the polarized GPD $\tilde{H}_{1}(x, 0,0)$ is related to $g_{1}(x)[8,15]$. It is believed that the $g_{1}$ gives the information of the polarized quark density, namely, the probability to find a polarized quark (with longitudinal momentum fraction $x$ ) parallel or antiparallel 
to the polarization of the target $[19,20]$. In addition, the sum $g_{T}=g_{1}+g_{2}$ involves the transverse spin density [19]. In general, the structure functions, $g_{2}$, or $g_{T}$, also receive the contributions from a quark-gluon correlation which comes from the twist-3 operator [21]. Thus, they may give the information of the "high-twist effects" in a system. Many theoretical and experimental studies have been preformed for both $g_{1}$ and $g_{2}$ (see, for example, Refs. [22-26]) in the literature. More details can be found in recent review articles [27-29].

To our knowledge, the spin-dependent structure functions $g_{1}$ and $g_{2}$ of spin- 1 hadrons, particular for the $\rho$ meson, have been rarely studied theoretically. Since we have successfully studied the unpolarized GPDs of the $\rho$ meson with a help of a light-front quark model, we extend our approach to further calculate the polarized GPDs of the $\rho$ meson, and try to obtain its $g_{1}(x)$ from the forward limit of the polarized GPDs $\tilde{H}_{1}(x, 0,0)$. It is known that the spin structure function $g_{2}$ is usually related to $g_{1}$ according to the Wandzura and Wilczek relation [30]. However, as emphasized by Jaffe and Ji [16,17], $g_{2}$ is not solely determined by $g_{1}$ as Wandzura and Wilczek concluded. There are another twist-2 function $\left(h_{T}\right)$ and a twist-3 term which may also have non-negligible contributions to $g_{2}$ (see Refs. [16,17,21]). In this work, however, only twist- 2 operators are involved and we ignore $h_{T}$ and twist- 3 terms as many other theoretical calculations $[21,22]$ did for simplicity.

In addition, the axial form factors for the spin- 1 particle $\tilde{G}_{1,2}$ are seldom discussed due to no axial current in electromagnetic interaction. However, after taking into account the electroweak interaction which contains axial vector currents, the two form factors can be measured through the respond functions $W_{1,2,8}$ [31]. This phenomenon is similar to the nucleon (spin-1/2) case [32]. Therefore, the axial form factors become important when we study the electro-weak structure of the system, such as the parity violating in the electron-deuteron scattering [33]. Since the axial form factors relate to the sum rules of the polarized GPDs of the system, we may also estimate them according to our obtained polarized GPDs for the $\rho$ meson.

This paper is organized as follows. In Sec. II, the definitions and sum rules of the polarized GPDs and the structure function $g_{1}$ etc. are briefly presented. Moreover, the light-front quark model employed in this and our previous works is also shortly discussed in this section. In Sec. III, the evolution for the spin structure function $g_{1}$ is discussed. Section IV gives our numerical results for the polarized GPDs, the spin structure functions $g_{1}, g_{2}$ and the axial form factors of the $\rho$ meson. Section V is devoted to a short summary.

\section{POLARIZED GPDS AND OUR MODEL}

Figure 1 illustrates the process we are considering. The notations are [11]

$$
\begin{aligned}
& t=\Delta^{2}=\left(p^{\prime}-p\right)^{2}=\left(q-q^{\prime}\right)^{2}, \quad Q^{2}=-q^{2}, \\
& \xi=-\frac{\Delta \cdot n}{2 P \cdot n}=-\frac{\Delta^{+}}{2 P^{+}}, \quad|\xi|=\frac{\Delta^{+}}{2 P^{+}}, \quad(|\xi| \leq 1) \\
& x=\frac{k \cdot n}{P \cdot n}=\frac{k^{+}}{P^{+}}, \quad(-1 \leq x \leq 1),
\end{aligned}
$$

where $p$ and $p^{\prime}$ are the 4-momenta of the incoming and outgoing $\rho$ mesons, $P=\left(p^{\prime}+p\right) / 2, \Delta=p^{\prime}-p, n$ is a lightlike 4-vector with $n^{2}=0$. Here $q$ is the virtual photon momentum, and $q^{\prime}$ is treated as a real one.

The four polarized GPDs, for a spin-1 particle, are introduced in Ref. [8],

$$
\begin{aligned}
\left.\frac{1}{2} \int \frac{d \lambda}{2 \pi} e^{i x(P z)}\left\langle p^{\prime}\left|\bar{q}\left(-\frac{1}{2} z\right) \not h \gamma_{5} q\left(\frac{1}{2} z\right)\right| p\right\rangle\right|_{z=\lambda n} \\
=-i \frac{\epsilon_{\mu \alpha \beta \gamma} n^{\mu} \epsilon^{\prime * \alpha} \epsilon^{\beta} P^{\gamma}}{P n} \tilde{H}_{1}^{q}(x, \xi, t) \\
\quad+2 i \frac{\epsilon_{\mu \alpha \beta \gamma} n^{\mu} \Delta^{\alpha} P^{\beta}}{P n} \frac{\epsilon^{\gamma}\left(\epsilon^{\prime *} P\right)+\epsilon^{\prime * \gamma}(\epsilon P)}{M^{2}} \tilde{H}_{2}^{q}(x, \xi, t) \\
+2 i \frac{\epsilon_{\mu \alpha \beta \gamma} n^{\mu} \Delta^{\alpha} P^{\beta}}{P n} \frac{\epsilon^{\gamma}\left(\epsilon^{\prime *} P\right)-\epsilon^{\prime * \gamma}(\epsilon P)}{M^{2}} \tilde{H}_{3}^{q}(x, \xi, t) \\
+\frac{i}{2} \frac{\epsilon_{\mu \alpha \beta \gamma} n^{\mu} \Delta^{\alpha} P^{\beta}}{P n} \frac{\epsilon^{\gamma}\left(\epsilon^{\prime *} n\right)+\epsilon^{\prime * \gamma}(\epsilon n)}{P n} \tilde{H}_{4}^{q}(x, \xi, t),
\end{aligned}
$$

where $\epsilon_{0123}=1$ and $M$ is the $\rho$ meson mass. Without loss of generality, we choose the $\rho^{+}$meson in this work and omit the superscript hereafter when there is no ambiguity. Thus, in the constituent quark model, only $u$ and $\bar{d}$ contribute to the current operator in Eq. (2). Time reversal constraints that $\tilde{H}_{3}^{q}$ are $\xi$-odd and all other GPDs $\xi$-even. Taking the lowest moments of the polarized GPDs in $x$, one recovers the axial vector form factors for each flavor $q[8]$,

$$
\int_{-1}^{1} d x \tilde{H}_{i}^{q}(x, \xi, t)=\tilde{G}_{i}^{q}(t), \quad(i=1,2)
$$

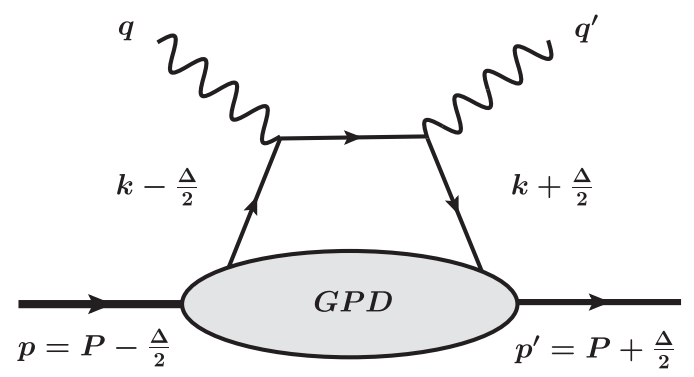

FIG. 1. The s-channel handbag diagram for GPDs. The u-channel one can be obtained by $q \leftrightarrow q^{\prime}$. 
with matrix elements of

$$
\begin{aligned}
\left\langle p^{\prime}\left|\bar{q}(0) \gamma^{\mu} \gamma_{5} q(0)\right| p\right\rangle & \\
= & -2 i \epsilon^{\mu}{ }_{\alpha \beta \gamma} \epsilon^{\prime * \alpha} \epsilon^{\beta} P^{\gamma} \tilde{G}_{1}^{q}(t) \\
& +4 i \epsilon^{\mu}{ }_{\alpha \beta \gamma} \Delta^{\alpha} P^{\beta} \frac{\epsilon^{\gamma}\left(\epsilon^{\prime *} P\right)+\epsilon^{\prime * \gamma}(\epsilon P)}{M^{2}} \tilde{G}_{2}^{q}(t) .
\end{aligned}
$$

For other two GPDs, time reversal invariance gives

$$
\int_{-1}^{1} d x \tilde{H}_{3}^{q}(x, \xi, t)=0
$$

and the Lorenz invariance constraints

$$
\int_{-1}^{1} d x \tilde{H}_{4}^{q}(x, \xi, t)=0
$$

With respect to the axial-vector current $J^{5 \mu}$, one gets the axial vector form factors

$$
\tilde{G}_{i}=\tilde{G}_{i}^{u}-\tilde{G}_{i}^{d}-\tilde{G}_{i}^{s}+\cdots, \quad(i=1,2),
$$

where the definition for individual flavor is given in Eq. (4). As shown later [in Eq. (15)], under the isospin symmetry, $\tilde{G}_{i}^{u}=\tilde{G}_{i}^{d}$ in $\rho^{+}$and the contributions of light $u$ and $d$ quarks to the total axial vector form factors cancel each other. When considering only the $u$ and $d$ flavours simultaneously, one gets $\tilde{G}_{1,2}=0$ [31].

Due to the isospin symmetry and charge symmetry (G-parity), the polarized (or axial) GPDs are related by

$$
\tilde{H}_{i, \rho^{+}}^{u}(x, \xi, t)=\tilde{H}_{i, \rho^{+}}^{d}(-x, \xi, t),
$$

where $i=1 \sim 4$. Project the axial (polarized) GPDs onto isoscalar and isovector combinations, we have

$$
\begin{aligned}
\tilde{H}_{i}^{I=0}(x, \xi, t) & =\frac{1}{2}\left[\tilde{H}_{i}^{u}(x, \xi, t)+\tilde{H}_{i}^{d}(x, \xi, t)\right], \\
\tilde{H}_{i}^{I=1}(x, \xi, t) & =\frac{1}{2}\left[\tilde{H}_{i}^{u}(x, \xi, t)-\tilde{H}_{i}^{d}(x, \xi, t)\right],
\end{aligned}
$$

and the corresponding axial vector isoscalar and isovector form factors are

$$
\begin{aligned}
& \int_{-1}^{1} d x \tilde{H}_{i}^{I=0}(x, \xi, t)=\tilde{G}_{i}^{u}(t)+\tilde{G}_{i}^{d}(t) \equiv \tilde{G}_{i}^{I=0}(t), \\
& \int_{-1}^{1} d x \tilde{H}_{i}^{I=1}(x, \xi, t)=\tilde{G}_{i}^{u}(t)-\tilde{G}_{i}^{d}(t) \equiv \tilde{G}_{i}^{I=1}(t) .
\end{aligned}
$$

With Eq. (8), one gets

$$
\begin{gathered}
\tilde{H}_{i}^{I=0}(x, \xi, t)=\tilde{H}_{i}^{I=0}(-x, \xi, t), \\
\tilde{H}_{i}^{I=1}(x, \xi, t)=-\tilde{H}_{i}^{I=1}(-x, \xi, t),
\end{gathered}
$$

which give

$$
\tilde{G}_{i}^{I=0}(t)=2 \tilde{G}_{i}^{u}(t), \quad \tilde{G}_{i}^{I=1}(t)=0, \quad(i=1,2),
$$

This results from $\tilde{G}_{i}^{u}=\tilde{G}_{i}^{d}$ in $\rho^{+}$.

For a comparison to the unpolarized case, we note that, for the unpolarized GPDs [11], there is an overall minus sign difference with respect to Eqs. (8) and (10), respectively,

$$
\begin{gathered}
H_{i, \rho^{+}}^{u}(x, \xi, t)=-H_{i, \rho^{+}}^{d}(-x, \xi, t), \\
H_{i, \rho^{+}}^{I=1}(x, \xi, t)=H_{i, \rho^{+}}^{I=1}(-x, \xi, t),
\end{gathered}
$$

where $i=1 \sim 5$. More details on the projection are referred to Refs. [11,34,35].

As emphasized in Ref. [31], the axial vector form factors $\tilde{G}_{1}$ and $\tilde{G}_{2}$ are usually discarded in the previous studies. After considering the electroweak interaction, one may expect nonzero strange quark contribution to $\tilde{G}_{1}$ and $\tilde{G}_{2}$, by measuring the difference between the cross sections of the pure electromagnetic interaction and the electroweak interaction. These measurements can provide an important probe for the electroweak structure of the nucleons [33]. For the $\rho$ meson, which is an isovector system, it is still quite interesting to know what these two form factors, for $u$ and $d$ flavors, look like under our phenomenological calculation.

In the forward limit $\Delta=0$, only $\tilde{H}_{1}^{q}$ survives and has quark density interpretation. Using the relation of the helicity amplitudes for finding a quark in a $\rho$ meson [8], one gets

$$
\tilde{H}_{1}^{q}(x, 0,0)=q_{\uparrow}^{1}(x)-q_{\downarrow}^{1}(x) \equiv \Delta q(x),
$$

where $x>0$ and $q_{\uparrow}^{1}(x)$ is the probability to find a quark with momentum fraction $x$ and polarization parallel to the $\rho$ meson helicity +1 . Here $\Delta q(x)$ is called the spin dependent density [1], or the polarized quark distribution [6]. The parity constraints $q_{\uparrow}^{1}=q_{\downarrow}^{-1}$. In the frame of GPDs, Eq. (18) with $x<0$ stands for the antiquark $(\bar{q})$ distribution at $-x$. This leads to the partonic decomposition $[1,6]$

$$
\tilde{H}_{1}^{q}(x, 0,0)=\theta(x) \Delta q(x)+\theta(-x) \Delta \bar{q}(-x) .
$$

By Eqs. (8) and (19), one gets

$$
\Delta u_{\rho^{+}}(x)=\Delta \bar{d}_{\rho^{+}}(x) .
$$

As discussed in Ref. [1], the $x$-even ("singlet") combination

$$
\tilde{H}_{1}^{q(+)}(x, \xi, t)=\tilde{H}_{1}^{q}(x, \xi, t)+\tilde{H}_{1}^{q}(-x, \xi, t)
$$

corresponds to the charge conjugation $C=+1$, and gives $\tilde{H}_{1}^{q(+)}(x, 0,0)=\Delta q(x)+\Delta \bar{q}(x)$ in the forward limit. The $x$-odd ("nonsinglet" or "valence") combination 


$$
\tilde{H}_{1}^{q(-)}(x, \xi, t)=\tilde{H}_{1}^{q}(x, \xi, t)-\tilde{H}_{1}^{q}(-x, \xi, t)
$$

corresponds to the charge conjugation $C=-1$, and gives $\tilde{H}_{1}^{q(-)}(x, 0,0)=\Delta q(x)-\Delta \bar{q}(x)$ in the forward limit. Thus, like the pion case $[35,36]$, for $\rho^{+}$, the valence (or nonsinglet) polarized quark distribution is

$$
\tilde{V}=\Delta u_{\rho}-\Delta \bar{u}_{\rho}+\Delta \bar{d}_{\rho}-\Delta d_{\rho},
$$

and the singlet polarized quark distribution is

$$
\tilde{S}=\Delta u_{\rho}+\Delta \bar{u}_{\rho}+\Delta d_{\rho}+\Delta \bar{d}_{\rho}+\Delta s_{\rho}+\Delta \bar{s}_{\rho} .
$$

These two combinations do not mix under evolution (see Sec. III). The sea-quark distribution is defined as [36]

$$
\tilde{s}=\tilde{S}-\tilde{V}=2\left(\Delta \bar{u}_{\rho}+\Delta d_{\rho}\right)+\Delta s_{\rho}+\Delta \bar{s}_{\rho} .
$$

In the present work, the $\rho^{+}$meson is restricted to be only composed by an active quark $u$ and an active antiquark $\bar{d}$, which means the contribution of sea quarks $(\bar{u}, d, s$ and $\bar{s})$ is not included here.

On the other hand, at leading order, the polarized structure function $g_{1}^{q}(x)$ gives the fraction of spin carried by quarks [15]

$$
g_{1}^{q}(x)=\frac{1}{2}\left[q_{\uparrow}^{1}(x)-q_{\downarrow}^{1}(x)\right]+\{q \rightarrow \bar{q}\},
$$

and follows the relation $[8,15]$

$$
g_{1}(x)=\sum_{q} e_{q}^{2} g_{1}^{q}(x)
$$

Therefore, with Eqs. (18) and (20), we get

$$
\begin{aligned}
g_{1}(x) & =\frac{1}{2} e_{u}^{2} \Delta u(x)+\frac{1}{2} e_{\frac{d}{d}}^{2} \Delta \bar{d}(x)=\frac{1}{2}\left(e_{u}^{2}+e_{\frac{2}{d}}^{2}\right) \Delta u(x), \\
\Delta q & \equiv \int_{0}^{1}\left[g_{1}^{u}(x)+g_{1}^{d}(x)\right] d x=\int_{0}^{1} \Delta u(x) d x .
\end{aligned}
$$

where $\Delta q$ is the total fraction of spin carried by valence $u$ and $\bar{d}$ in $\rho^{+}$.

In general, the rigorous expression for the structure function $g_{2}$ contains another twist-2 piece, "transversity" $h_{T}$, and a twist-3 piece arising from quark-gluon correlation $[21,22] . h_{T}$ is proportional to the ratio of the current quark mass to the target mass $\left(\sim m_{c} / M\right)$ and it is commonly neglected in most studies [22]. In present work, both $h_{T}$ and the twist- 3 parts are neglected, although it may not be small. Under those approximations, one gets the WandzuraWilcze relation [30] for $g_{2}$,

$$
g_{2}^{\mathrm{WW}}(x)=-g_{1}(x)+\int_{x}^{1} \frac{d y}{y} g_{1}(y) .
$$

Here, the $Q^{2}$-dependence is ignored, since at large $Q^{2}$, the $g_{1}$ and $g_{2}$ become scaling. It may not be a good approximation to identify $g_{2}(x)=g_{2}^{\mathrm{WW}}(x)$ (which may have $15 \sim$ $40 \%$ breaking of the size of $g_{2}$ [37]), however, we argue that it, at least, allows us to estimate the contribution of the axial current operator to $g_{2}$. In this case, it is easy to verify the Burkhardt-Cottingham sum rule [38] by changing the integral variables,

$$
\int_{0}^{1} g_{2}(x) d x=0 .
$$

Notes that, according to Ref. [16], this relation remains to be tested since the derivation in [38] is based on the assumption of the Regge theory. However, Ref. [22] claims, for proton, this sum rule for $g_{2}$ holds up to order of $O\left(M^{2} / Q^{2}\right)$. Finally, with those approximations, one gets the transverse spin density $[19,30]$

$$
g_{T}(x)=g_{1}(x)+g_{2}(x) \sim \int_{x}^{1} \frac{d y}{y} g_{1}(y) .
$$

The Mellin moment of a function $f(x)$ is defined as

$$
M_{n}(f)=\int_{0}^{1} x^{n-1} f(x) d x .
$$

For the $\rho$ meson case, at the leading order (twist 2), one finds [15]

$$
2 M_{n}\left(g_{1}^{q}\right)=C_{n}^{(1)} r_{n}
$$

where $C_{n}^{(k)}=1+O(\alpha)$ is the Wilson coefficient of the operator product expansion and $r_{n}$ are the reduced matrix elements. These relations hold for both even and odd $n$th orders with the quenched approximation. Note that there are two different sets of notations labeling the moments of $F_{1}, b_{1}$ and $g_{1}$ respectively in Refs. [15,20]. Here we follow the former.

In a numerical calculation, we employ the phenomenological light-front quark model to describe the interaction between the spin- $1 \rho$ meson and its constitutes $u$ and $d$. It is based on a effective interaction Lagrangian for the $\rho \rightarrow \bar{q} q$ vertex,

$$
\begin{aligned}
\mathcal{L}_{I} & =-\frac{\imath M}{f_{\rho}} \bar{q} \Gamma^{\mu} \tau q \cdot \rho_{\mu} \\
& =-\frac{l \sqrt{2} M}{f_{\rho}}\left[\frac{\bar{u} \Gamma^{\mu} u-\bar{d} \Gamma^{\mu} d}{\sqrt{2}} \rho_{\mu}^{0}+\bar{u} \Gamma^{\mu} d \rho_{\mu}^{+}+\bar{d} \Gamma^{\mu} u \rho_{\mu}^{-}\right],
\end{aligned}
$$




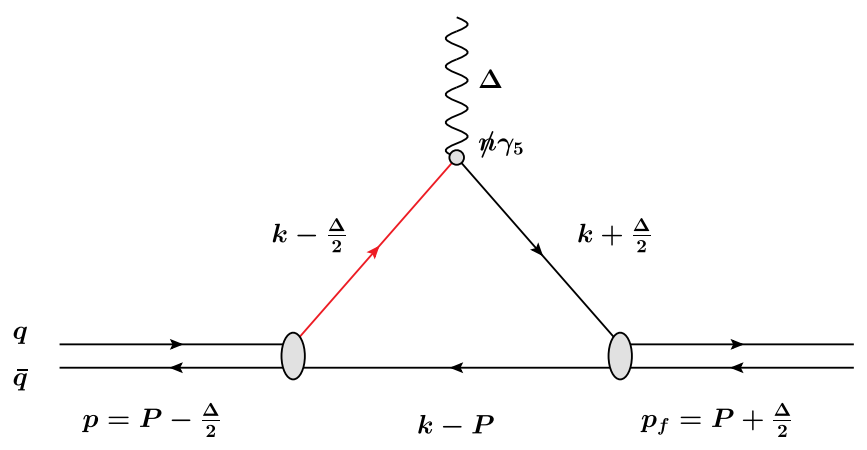

FIG. 2. The struck $u$ quark in the valence regime for axial current. The momentum of the red line have positive plus component.

where $\rho_{\mu}$ is the $\rho$ meson field, $f_{\rho}$ is the $\rho$ decay constant (which may be absorbed in the normalization factor $N$ ), and $\Gamma^{\mu}$ is a Bethe-Salpeter amplitude (BSA) [11,39],

$$
\Gamma^{\mu}=N \frac{\gamma^{\mu}-\left(k_{q}+k_{\bar{q}}\right)^{\mu} /\left(M_{i, f}+2 m\right)}{\left[k_{q}^{2}-m_{R}^{2}+\imath \epsilon\right]\left[k_{\bar{q}}^{2}-m_{R}^{2}+\imath \epsilon\right]},
$$

where, for the $u$ quark contribution, the struck $u$ quark momentum $k_{u}=k-\Delta / 2$ and the spectator constituent momentum is $k_{s}=k_{\bar{d}}=k-P$, as shown in Fig. $2 . N$ is the normalization constant, $m$ and $m_{R}$ are the constituent quark and the regulator masses, respectively, and $M_{i, f}$ are the kinematic invariant masses, [11,39]

$$
\begin{gathered}
M_{i}^{2}=\frac{\kappa_{\perp}^{2}+m^{2}}{1-x^{\prime}}+\frac{\kappa_{\perp}^{2}+m^{2}}{x^{\prime}}, \\
M_{f}^{2}=\frac{\kappa_{\perp}^{\prime 2}+m^{2}}{1-x^{\prime \prime}}+\frac{\kappa_{\perp}^{\prime 2}+m^{2}}{x^{\prime \prime}},
\end{gathered}
$$

where the subscript $i(f)$ for initial(final) state and, following momenta convention in Fig. 2, the LF momentum fractions $x^{\prime}\left(x^{\prime \prime}\right)$ and $\kappa_{\perp}\left(\kappa_{\perp}^{\prime}\right)$ are

$$
\begin{aligned}
x^{\prime} & =-\frac{k_{s}^{+}}{p^{+}}=\frac{1-x}{1-|\xi|}, & x^{\prime \prime} & =x^{\prime} \frac{p^{+}}{p^{\prime+}}=\frac{1-x}{1+|\xi|}, \\
\kappa_{\perp} & =(k-P)_{\perp}-\frac{x^{\prime}}{2} \Delta_{\perp}, & \kappa_{\perp}^{\prime} & =(k-P)_{\perp}+\frac{x^{\prime \prime}}{2} \Delta_{\perp} .
\end{aligned}
$$

In the nonvalence regime where $-|\xi|<x<|\xi|$ leads to $x^{\prime}>1$ in Eq. (36) and (38), and the initial vertex becomes the non-wave function vertex. To keep the mass square positive, as Refs. [11,39], we directly replace $1-x^{\prime}$ with $x^{\prime}-1$ in Eq. (36) and get

$$
M_{i(N V)}^{2}=\frac{\kappa_{\perp}^{2}+m^{2}}{x^{\prime}-1}+\frac{\kappa_{\perp}^{2}+m^{2}}{x^{\prime}} .
$$

Here, to keep this phenomenological $\Gamma^{\mu}$ respecting to the isospin symmetry [which is required by Eqs. (8), (16) and (20)], one has to employ the symmetric momenta convention as shown in Fig. 2. More details are explained in our previous work [11].

The expressions for individual axial GPDs can be obtained through the same way showed in the Appendix of Ref. [11]. For example, the $\tilde{H}_{1}^{u}$ is

$$
\begin{aligned}
\tilde{H}_{1}^{u}(x, \xi, t)= & N_{\mu \nu} \int \frac{d^{4} k}{(2 \pi)^{4}} \delta[n \cdot(x P-k)] \\
& \times \operatorname{Tr}\left[\frac{\imath(\not k-P+m)}{(k-P)^{2}-m^{2}+\imath \epsilon} \Gamma^{\nu} \frac{l\left(\not k+\frac{\not}{2}+m\right)}{\left(k+\frac{\Delta}{2}\right)^{2}-m^{2}+\imath \epsilon} \not h \gamma_{5}\right. \\
& \left.\times \frac{\imath\left(\not k-\frac{\not}{2}+m\right)}{\left(k-\frac{\Delta}{2}\right)^{2}-m^{2}+\imath \epsilon} \Gamma^{\mu}\right],
\end{aligned}
$$

where

$$
N_{\mu \nu}=i \frac{M^{2}}{f_{\rho}^{2}} \frac{c^{2} n^{\alpha} P^{\beta} \epsilon_{\alpha \beta \mu \nu}}{4(2 \pi)^{3} \sqrt{\omega_{p^{\prime}} \omega_{p}}(P \cdot n)},
$$

with $c$ being a normalization factor.

\section{ON THE QCD EVOLUTION}

Comparing the model-dependent results to the available "data," like the Lattice QCD calculation, one may perform a QCD evolution to evolve the parton distribution and its moments from the factorization scale $\mu_{0}$ to the scale that a lattice QCD calculation is performed. For the calculated $\rho$ meson polarized GPDs or structure functions in the present work, we compare our result with the Lattice QCD results at the scale $\mu=2.4 \mathrm{GeV}$ with quenched approximation [15], as our previous work for the unpolarized ones. Here, we ignore the gluon contribution to the evolution, thus, we can adopt the same (LO) DGLAP evolution function for the moments of the single flavor structure function $g_{1}^{u}(x)$ as

$$
\frac{\tilde{V}_{n}^{u}(\mu)}{\tilde{V}_{n}^{u}\left(\mu_{0}\right)}=\left(\frac{\alpha(\mu)}{\alpha\left(\mu_{0}\right)}\right)^{\gamma_{n}^{(0)} /\left(2 \beta_{0}\right)},
$$

where the single quark spin fractions

$$
\tilde{V}_{n}^{u}=2 M_{n+1}\left[g_{1}^{u}(x)\right] \sim r_{n+1}
$$

and the running coupling constant is

$$
\alpha(\mu)=\frac{4 \pi}{\beta_{0} \log \left(\mu^{2} / \Lambda_{\mathrm{QCD}}^{2}\right)},
$$

where $\beta_{0}=11 N_{c} / 3-2 N_{f} / 3$ with $N_{c}=N_{f}=3$ and

$$
\Lambda_{\mathrm{QCD}}=0.226 \mathrm{GeV}
$$

being employed [36,40]. In our previous work, we performed the evolution of the Mellin moments of unpolarized 
structure function, and found the factorization scale of the model is $\mu_{0}=528_{-62}^{+77} \mathrm{MeV}$.

In our previous work, we obtained the evolution ratio for the valence quark distribution, by calculating the evolution of the active $u$ quark unpolarized distribution. Here we adopt the same ratio for the evolution of valence polarized quark distribution (or their Mellin moments) to compare with the lattice QCD results since the scale $(\mu=2.4 \mathrm{GeV})$ is same for both unpolarized and polarized cases. In addition, the sea quark contributions [Eq. (25)] are excluded from our calculation, thus one can observe that the nonsinglet [Eq. (23)] and singlet [Eq. (24)] polarized quark distributions make no more difference in present work.

\section{NUMERICAL RESULTS}

Following our previous work on the unpolarized GPDs [11], we take the two model parameters, the constituent mass $m=0.403 \mathrm{GeV}$ and regulator mass $m_{R}=1.61 \mathrm{GeV}$. We simply extend the model to the polarized GPDs $\tilde{H}_{1,2}$ case. Their $x$ - and $t$-dependences with skewness $\xi=0$ and $\xi=-0.4$ are shown in Figs. 3 and 4, respectively. The results are normalized with respect to the corresponding $u$ quark axial form factors. The obtained polarized GPDs have opposite values in the region $-1<x<0$ with respect to the region $0<x<1$ at the same $t$, as a consequence of the isospin symmetry of our model. At the joint points of valance and nonvalance regions, namely at $|x|=|\xi|=0.4$ in Figs. 3(b) and 4(b), our resulted $\tilde{H}_{1,2}$ are continuous. This phenomenon fulfills the requirement of the consistency of the factorization at leading twist [1]. Here, we take the momentum transfer $t$ up to $-10 \mathrm{GeV}^{2}$, similar to the unpolarized case. Comparing to the unpolarized GPDs, especially $H_{1}$, we find that the polarized GPDs $\tilde{H}_{1,2}$ vary much slow with respect to $t$. Figures 5 and 6 show the

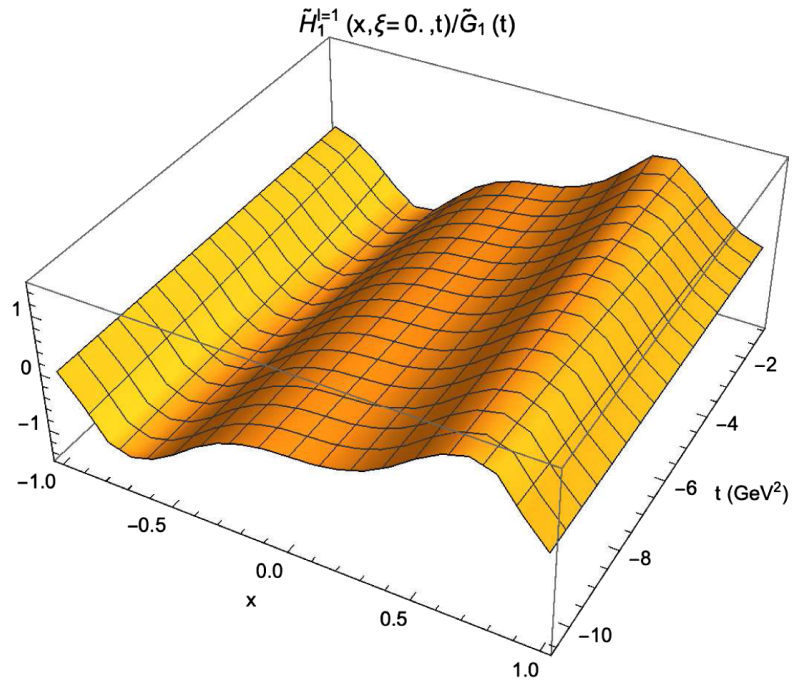

(a) $\xi=0$ single flavor axial form factor $\tilde{G}_{1}^{u}(t)$ and $\tilde{G}_{2}^{u}(t)$, respectively. Within the region $-10 \mathrm{GeV}^{2}<t<0, \tilde{G}_{1}^{u}(t)$ is larger than $\tilde{G}_{2}^{u}(t)$ and decreases slower than $\tilde{G}_{2}^{u}(t)$ as $t$ increases. The starting points are $\tilde{G}_{1}^{u}(0)=0.86$ and $\tilde{G}_{2}^{u}(0)=-0.16$, respectively. Correspondingly, we have $\tilde{G}_{1}^{I=0}(0)=1.72$ and $\tilde{G}_{2}^{I=0}(0)=-0.32$, respectively.

In Figs. 7 and 8, the $x$ dependence of $g_{1}^{u}$ and $g_{2}^{u}$ are shown. Our result for $g_{1}^{u}(x)$ remains positive in the whole $0<x<1$ region and it is nearly symmetry around $x=1 / 2$. The available experimental data for deuteron $g_{1}^{(d)}(x)$, summarized in Ref. [25], have negative values at small $x$ region, but it is believed to be consistent with zero after combining the new COMPASS result [26]. We think our result for the $\rho$ meson may indirectly confirm the positiveness of $g_{1}(x)$. In general, our twist-2 results for the $\rho$ meson $g_{1}$ have similar $x$-dependence behavior with the $g_{1}$ of the deuteron in the new COMPASS result [26] (see its Fig. 4). Summing over $x$ of $g_{1}^{u}(x)+g_{1}^{d}(x)$ as Eq. (29), we get

$$
\Delta q=0.86
$$

which means the fraction of spin carried by the constituent quark and antiquark in $\rho$ meson is 0.86 , while the expected value is 1 . This result is similar to the case of the nucleon (see for example Ref. [41]). In general, the total fraction of spin carried by quarks and antiquarks in nucleon is not more than $30 \%$ to $50 \%$. It is well known as the "spin crisis" issue (or "spin puzzle") [6,27,29,41]. As proposed by Sehgal [42], another important contribution to the proton spin may come from the orbital angular momentum of partons. Through the light-cone representation of the spin and orbital angular momentum of relativistic composite systems, Brodsky, Hwang, Ma and Schmidt [43]

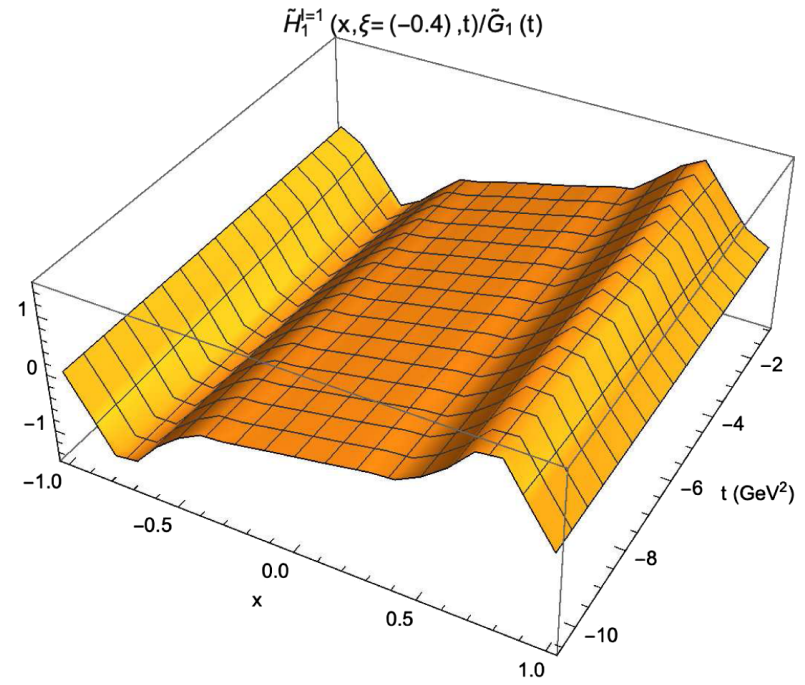

(b) $\xi=-0.4$

FIG. 3. $\rho^{+}$GPD $\tilde{H}_{1}$ with $\xi=0$ and -0.4 . 


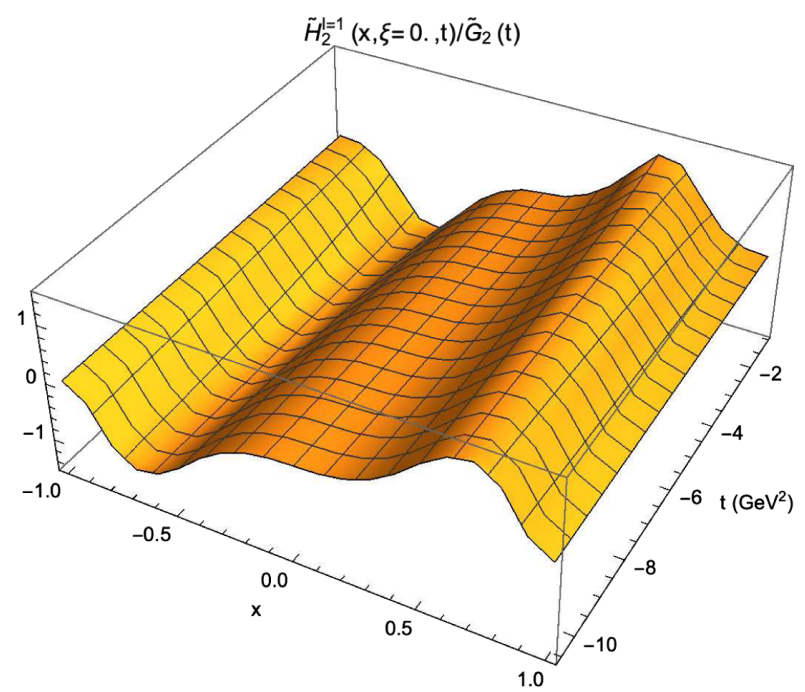

(a) $\xi=0$

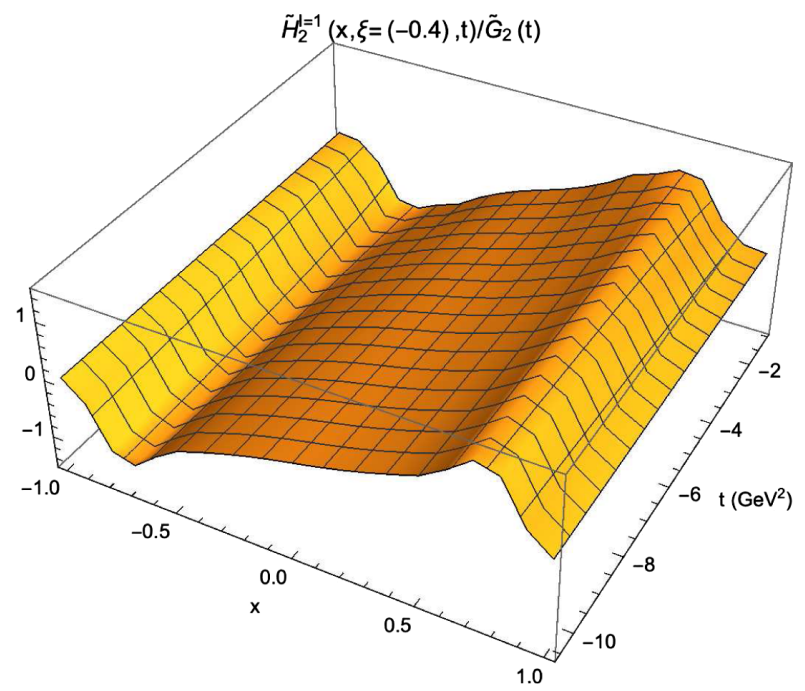

(b) $\xi=-0.4$

FIG. 4. $\rho^{+}$GPD $\tilde{H}_{2}$ with $\xi=0$ and -0.4 .

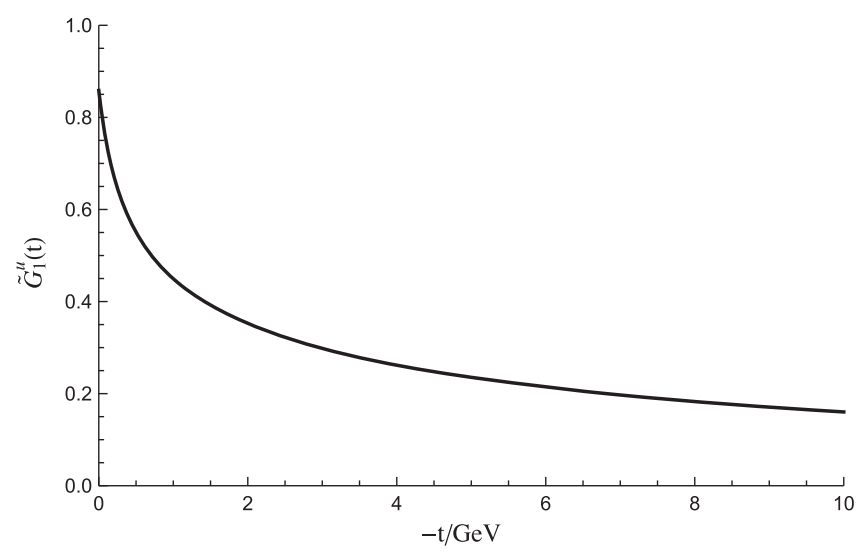

FIG. 5. The $u$ quark axial form factor $\tilde{G}_{1}^{u}(t)$.

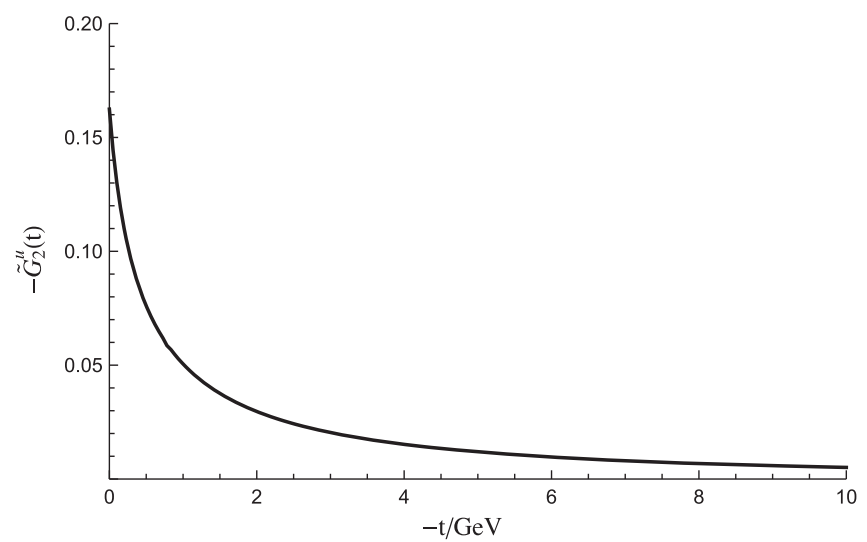

FIG. 6. The $u$ quark axial form factor $\tilde{G}_{2}^{u}(t)$.

have shown that the "spin crisis" of the nucleon can be explained due to the relativistic motion of quarks, and the contribution of the orbital angular momentum. Thus the small $\Delta q$ can be naturally understood. According to

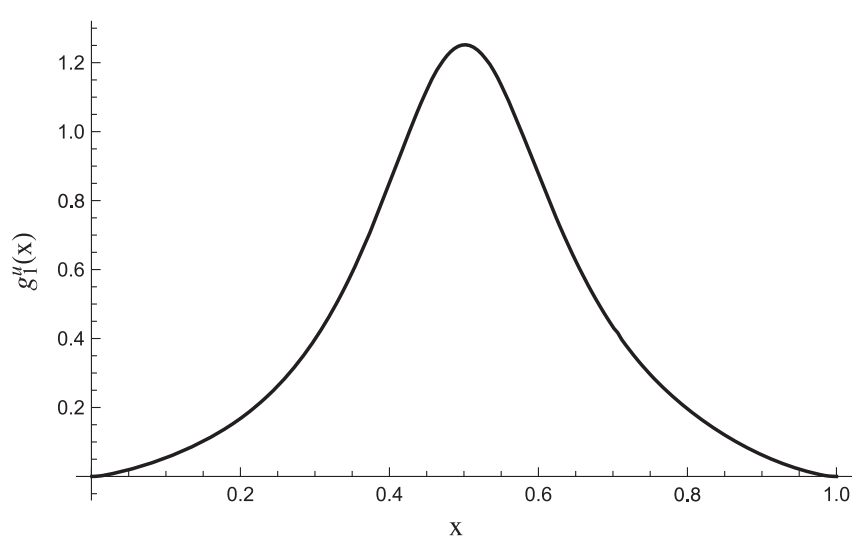

FIG. 7. The $u$ quark structure function $g_{1}^{u}(x)$.

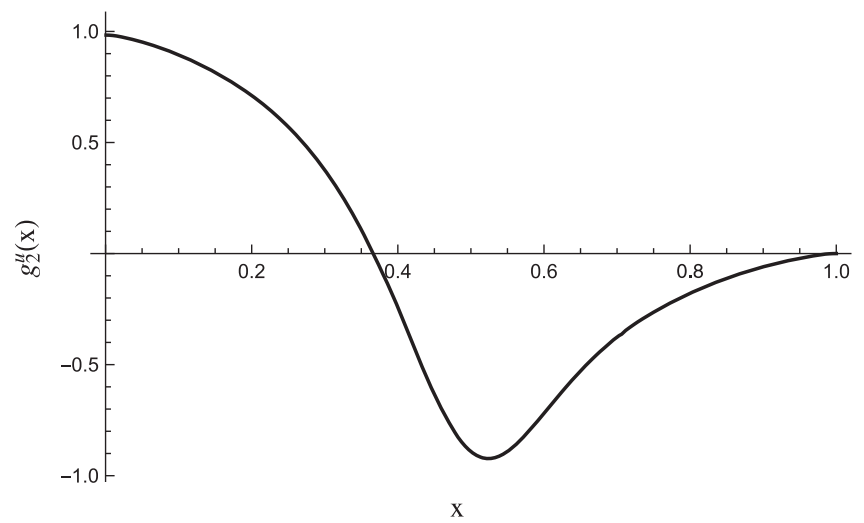

FIG. 8. The $u$ quark structure function $g_{2}^{u}(x)$.

Refs. [44,45], the nucleon "spin crisis" maybe also be understood through the pion cloud effect together with relativistic corrections and one-gluon exchange, which can transfer the quark spin to the orbital angular momentum 


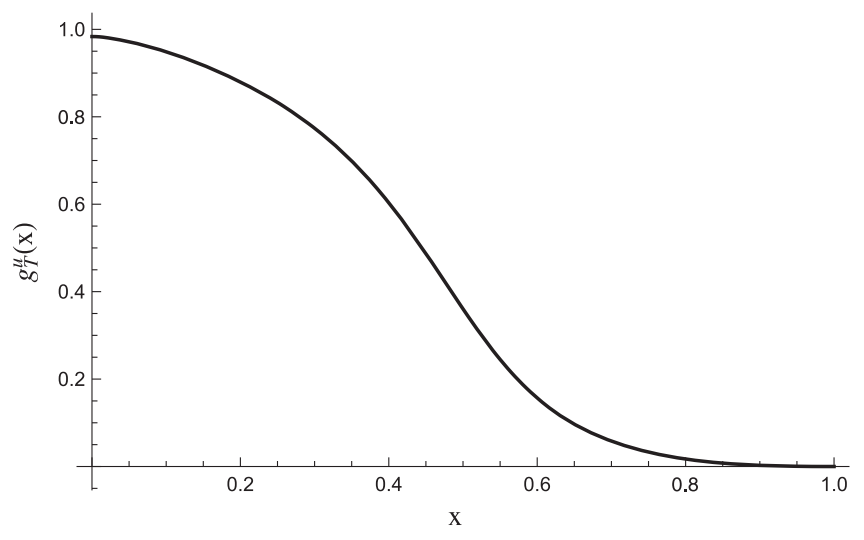

FIG. 9. The $u$ quark transverse spin density $g_{T}^{u}(x)$.

and it mainly accounts for the missing spin. The pions play a role of quark and antiquark sea. Here, we suggest that the orbital angular momentum may also be an important source for the $\rho$ meson spin and the corresponding parton splitting processes $q \rightarrow q g$ and $g \rightarrow q \bar{q}$ responsible for the DGLAP evolution, generate the orbital angular momentum [1]. After the evolution to a higher scale $\mu=2.4 \mathrm{GeV}$, as $r_{1}$ shown in Fig. 10 later, $\Delta q$ becomes to around $60 \%$.

Another way to understand the proton spin problem (see for example Refs. [46,47]) is to consider the Wigner rotation of the spin of a moving quark. In this sense, there is no need to require the sum of quark's spin equals the total proton spin in the light front frame.

For the $g_{2}(x)$ structure function, the present constituent model predicts that

$$
\int_{0}^{1} g_{2}(x) d x=0.000112
$$

comparing with the Burkhardt-Cottingham sum rule Eq. (31), we conclude that it is numerically consistent with vanishing. With Eq. (31), we find that $g_{2}(x)$ has a remarkable feature of a nontrivial zero between $x=0$ and $x=1$. Note again that $g_{2}$ should also receives contributions from twist-3 quark-gluon correlation which may be not small comparing to that of the twist-2 piece. The importance of this unique feature has stressed in previous works [17,21,24].

If one takes the massless limit of quark (asymptotic free), then $g_{T}=g_{1}+g_{2}$ would be small, but this phenomenon contradicts to the $\rho$ meson rest mass, since the quarks are not free inside hadrons, especially in the constituent quark model. Our results (see Fig. 9) tells that $g_{T}^{u}$ is sizeable in the small and moderate $x$ regions $(<0.5)$ and becomes much smaller in large $x$ region. It may be interpreted that as the quark possesses more fraction of longitudinal momentum (larger $x$ ), it contributes less to the transverse spin density.

The numerical evolution for the polarized structure functions is similar to the unpolarized case. With the same ratio, which is 0.67 , we evolute our results for the moments of $g_{1}$ to the scale of the lattice QCD result [15]. We compare the results of the two approaches in Fig. 10. The results of $r_{n}$ in Ref. [15] was obtained with two sets of operators, and

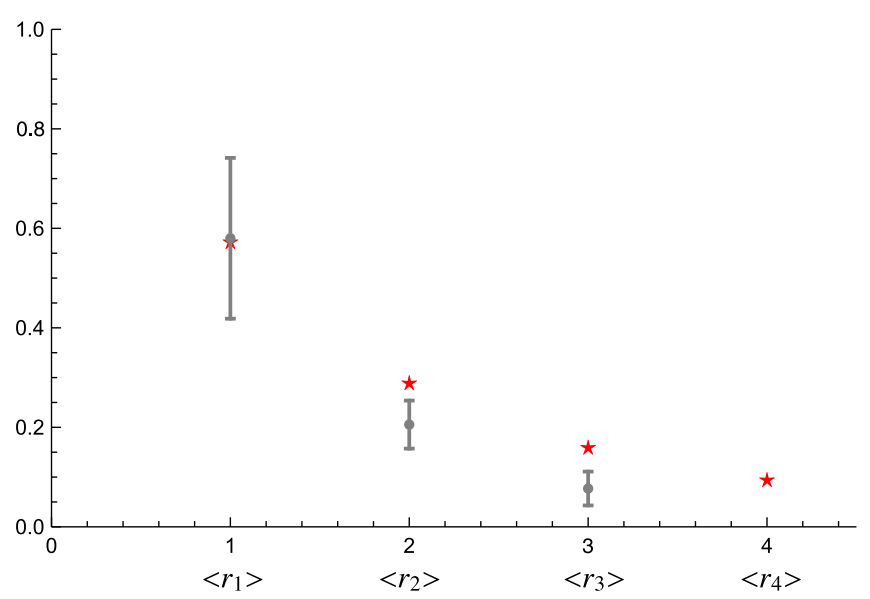

FIG. 10. $r_{n}$ for u quark. The red stars are our results and the gray ones with errors are the lattice QCD results [15].

in Fig. 10 we plot the averaged values. In general, our results agree with the lattice QCD ones. Moreover, one more order of the moment (see $r_{4}$ ) is given by our calculation.

\section{CONCLUSIONS}

In this work, we extend our previous work on the $\rho$ meson GPDs with the light-front constituent quark model to the polarized case. The polarized GPDs $\tilde{H}_{1,2}$ with nonzero skewness (e.g., $\xi=-0.4$ ) are given in 3-D plots with respect to $x$ and $t$. With the sum rules for $\tilde{H}_{1,2}$, we obtained the axial form factors $\tilde{G}_{1,2}$, the spin structure functions $g_{1}(x)$ and $g_{2}(x)$, and the moments for $g_{1}(x)$. After the evolution, our results of the moments of $g_{1}$ agree with the Lattice QCD results. The quark spin contribution $(\Delta q=0.86)$ to the $\rho$ meson spin and the transverse spin density $g_{T}$ for the $\rho$ meson are also estimated with the constituent quark model for the first time. The small value of $\Delta q$ for $\rho$ may be mainly explained by its transfer to the orbital angular momentum carried by valence quarks, which is also a possible resolution of the nucleon spin problem. Our numerical result for $g_{2}(x)$ shows that the Burkhardt-Cottingham sum rule holds reasonably well in this work.

\section{ACKNOWLEDGMENTS}

One of the authors (B.D.S.) acknowledges RuhrUniversität Bochum, for the warm hospitality where part of this work is being done; and thanks Bernard Pire and Maxim V. Polyakov for helpful discussions. This work is supported by the National Natural Science Foundation of China under Grant No. 11475192, by the fund provided to the Sino-German CRC 110 "Symmetries and the Emergence of Structure in QCD" project by the NSFC under Grant No. 11621131001, and the Key Research Program of Frontier Sciences, CAS, Grant No. Y7292610K1 and the State Scholarship Fund of China Scholarship Council No. 201804910428 and the DAAD Research Grants 2018 No. 57381332. 
[1] M. Diehl, Phys. Rep. 388, 41 (2003).

[2] M. Burkardt, Int. J. Mod. Phys. A 18, 173 (2003).

[3] G. A. Miller, Annu. Rev. Nucl. Part. Sci. 60, 1 (2010).

[4] B.-D. Sun and Y.-B. Dong, Chin. Phys. C 42, 063104 (2018).

[5] X. D. Ji, Phys. Rev. D 55, 7114 (1997).

[6] X. D. Ji, J. Phys. G 24, 1181 (1998).

[7] P. Hoodbhoy, R. L. Jaffe, and A. Manohar, Nucl. Phys. B312, 571 (1989).

[8] E. R. Berger, F. Cano, M. Diehl, and B. Pire, Phys. Rev. Lett. 87, 142302 (2001).

[9] W. Cosyn, Y.-B. Dong, S. Kumano, and M. Sargsian, Phys. Rev. D 95, 074036 (2017).

[10] W. Cosyn and B. Pire, Phys. Rev. D 98, 074020 (2018).

[11] B.-D. Sun and Y.-B. Dong, Phys. Rev. D 96, 036019 (2017).

[12] J. P. B. C. de Melo and T. Frederico, Phys. Rev. C 55, 2043 (1997).

[13] D. G. Gudino and G. T. Schnchez, Int. J. Mod. Phys. Conf. Ser. 35, 1460463 (2014).

[14] A. F. Krutov, R. G. Polezhaev, and V. E. Troitsky, Phys. Rev. D 97, 033007 (2018).

[15] C. Best, M. Gockeler, R. Horsley, E. M. Ilgenfritz, H. Perlt, P. Rakow, A.Schafer, G. Schierholz, A. Schiller, and S. Schramm, Phys. Rev. D 56, 2743 (1997).

[16] R. L. Jaffe, Comments Nucl. Part. Phys. 19, 239 (1990).

[17] R. L. Jaffe and X. D. Ji, Phys. Rev. D 43, 724 (1991).

[18] P. A. M. Guichon and M. Vanderhaeghen, Prog. Part. Nucl. Phys. 41, 125 (1998).

[19] R. P. Feynman, Photon-Hadron Interactions (Benjamin Press, New York, 1972), pp. 132-159.

[20] L. Mankiewicz and Z. Ryzak, Phys. Rev. D 43, 733 (1991).

[21] J. L. Cortes, B. Pire, and J. P. Ralston, Z. Phys. C 55, 409 (1992).

[22] X. Song, Phys. Rev. D 54, 1955 (1996).

[23] Y.-B. Dong, Phys. Lett. B 408, 393 (1997).

[24] P. L. Anthony et al., Phys. Lett. B 458, 529 (1999); 553, 18 (2003).

[25] A. Airapetian et al., Phys. Rev. D 75, 012007 (2007).
[26] C. Adolph et al., Phys. Lett. B 769, 34 (2017).

[27] S. E. Kuhn, J.-P. Chen, and E. Leader, Prog. Part. Nucl. Phys. 63, 1 (2009).

[28] J. P. Chen, Int. J. Mod. Phys. E 19, 1893 (2010).

[29] C. A. Aidala, S. D. Bass, D. Hasch, and G. K. Mallot, Rev. Mod. Phys. 85, 655 (2013).

[30] S. Wandzura and F. Wilczek, Phys. Lett. B 72, 195 (1977).

[31] S. J. Pollock, Phys. Rev. D 42, 3010 (1990); 43, 2447(E) (1991).

[32] D. B. Kaplan and A. Manohar, Nucl. Phys. B310, 527 (1988).

[33] T. M. Ito et al., Phys. Rev. Lett. 92, 102003 (2004).

[34] T. Frederico, E. Pace, B. Pasquini, and G. Salme, Phys. Rev. D 80, 054021 (2009).

[35] M. V. Polyakov and C. Weiss, Phys. Rev. D 60, 114017 (1999).

[36] W. Broniowski, E. R. Arriola, and K. Golec-Biernat, Phys. Rev. D 77, 034023 (2008).

[37] A. Accardi, A. Bacchetta, W. Melnitchouk, and M. Schlegel, J. High Energy Phys. 11 (2009) 093.

[38] H. Burkhardt and W. N. Cottingham, Ann. Phys. (N.Y.) 56, 453 (1970).

[39] H.-M. Choi and C.-R. Ji, Phys. Rev. D 70, 053015 (2004).

[40] W. Broniowski and E. R. Arriola, Phys. Rev. D 78, 094011 (2008).

[41] A. Deur, S. J. Brodsky, and G. F. De Téramond, arXiv:1807 .05250 .

[42] L. M. Sehgal, Phys. Rev. D 10, 1663 (1974); 11, 2016(E) (1975).

[43] S. J. Brodsky, D. S. Hwang, B. Q. Ma, and I. Schmidt, Nucl. Phys. B593, 311 (2001).

[44] A. W. Schreiber and A. W. Thomas, Phys. Lett. B 215, 141 (1988).

[45] F. Myhrer and A. W. Thomas, Phys. Lett. B 663, 302 (2008).

[46] B. Q. Ma, J. Phys. G 17, L53 (1991).

[47] B. Q. Ma and Q. R. Zhang, Z. Phys. C 58, 479 (1993). 